\title{
Scrub Typhus: An Emerging Etiology among Undiagnosed Febrile Cases in Western Part of Uttar Pradesh, India
}

\author{
Asfia Sultan $^{1 *}$ (D) Afaf Shuaib $^{1}$ (D) Meher Rizvi $^{1}$ (D) Fatima Khan $^{1}$ (D), \\ Rama Chaudhry ${ }^{2}$ a and Chandan Kumar Thakur ${ }^{2}$ iD \\ ${ }^{1}$ Department of Microbiology, Jawaharlal Nehru Medical College and Hospital, \\ Aligarh Muslim University, Aligarh - 202 002, Uttar Pradesh, India. \\ ${ }^{2}$ Department of Microbiology, All India Institute of Medical Science, New Delhi - 110 029, India.
}

\begin{abstract}
Scrub typhus is a re-emerging zoonotic rickettsial disease and is prevalent in many parts of India. Scrub typhus in Uttar Pradesh is virtually unknown but has shown emergence in recent reports. In this context, a prospective study was planned to analyze the prevalence and clinic-epidemiological parameters of scrub typhus in febrile patients present in JNMC hospital. Patients of $>15$ years with undiagnosed acute febrile illness of more than $\mathbf{5}$ days with or without acute respiratory failure and acute renal failure (ARF) were included in the study. Clinical profile was recorded through predesigned proforma. Blood samples were collected and serological tests were performed. PCR was performed on whole blood samples of positive patients. Total $\mathbf{4 1 4}$ patients, who were negative for other common febrile etiologies, i.e.: malaria, dengue, enteric fever were included in the study. Out of 414 patients, 112 (27\%) were found positive for scrub typhus by ELISA and micro-immunofluorescence (MIF). PCR was negative in all cases. Slight male predominance $(52.6 \%)$ was observed. Significant number of positive cases $90(80.3 \%)$ were between $18-46$ years of age ( $P$ value $<0.05)$. Majority of cases appeared during winter season Respiratory involvement was significant in positive cases ( $P$ value $<0.05$ ). Among the gastro-intestinal symptoms, diarrhea and vomiting were also significant $(P$ value $<0.005)$. All the patients responded to the treatment. No complications and mortality was observed. Scrub typhus should be considered in undiagnosed febrile patients coming from rural and agricultural background with respiratory and gastrointestinal symptoms. Serology is reliable in the diagnosis of scrub typhus as molecular testing is costly, labor intensive and not helpful.
\end{abstract}

Keywords: Scrub typhus, micro-immunofluorescence, ELISA, undifferentiated fever

*Correspondence: drasfia@gmail.com

(Received: September 14, 2021; accepted: January 4, 2022)

Citation: Sultan A, Shuaib A, Rizvi M, Khan F, Chaudhry R, Thakur CK. Scrub Typhus: An Emerging Etiology among Undiagnosed Febrile Cases in Western Part of Uttar Pradesh, India. J Pure Appl Microbiol. 2022;16(1):606-612. doi: 10.22207/JPAM.16.1.60

(C) The Author(s) 2022. Open Access. This article is distributed under the terms of the Creative Commons Attribution 4.0 International License which permits unrestricted use, sharing, distribution, and reproduction in any medium, provided you give appropriate credit to the original author(s) and the source, provide a link to the Creative Commons license, and indicate if changes were made. 


\section{INTRODUCTION}

Scrub typhus is a zoonotic rickettsial illness caused by Orientia tsutsugamushi and is known since World War II. ${ }^{1}$ It is considered as a re-emerging disease and prevalent in many parts of India. ${ }^{2}$ It is characterized by non-specific, flu like illness of fever, headache and myalgias with or without systemic involvement. Scrub typhus is one of the world's most under diagnosed / under reported diseases according to World Health Organization. ${ }^{3}$ One reason for its low index of suspicion is the prevalence of other febrile diseases like dengue, malaria, leptospira etc with overlapping clinical manifestations in the postmonsoon period. ${ }^{4}$

Early symptoms are non specific and difficult to distinguish from other diseases but serious and fatal complications are not uncommon which include pneumonia, acute respiratory distress syndrome (ARDS), myocarditis, meningoencephalitis, acute renal failure and gastrointestinal bleeding. ${ }^{5}$ It threatens one billion people globally, and causes illness in one million people each year. ${ }^{6}$ Early diagnosis is therefore important for timely antimicrobial therapy which may prevent complications.

Indian studies have documented a prevalence rate of around 33.3\%. ${ }^{7}$ However, the available data in India is an underestimate of the true rates, due to lack of community based data and non-availability of confirmatory laboratory tests. ${ }^{8}$

Scrub typhus in India has been documented from several states: Karnataka, Tamil Nadu New Delhi, Jammu Kashmir, Himachal Pradesh, Utta-rakhand. ${ }^{4,9-12}$ Despite various reports from all over India, there is no documentation of scrub typhus from western Uttar Pradesh on significant sample size. Our previous report was a pilot study done over a short period of time. ${ }^{13}$ Given the broad prevalence of scrub typhus in India and variations in clinical manifestations due to strain variation, thorough investigation into the regional variation in clinical presentation and strains is required.

The present study was done to document the prevalence of Scrub typhus among febrile patients reported to hospital in western Uttar Pradesh, in relation to clinical manifestations and strain positivity among diagnosed patients.

\section{MATERIALS AND METHODS}

The study was conducted in the Department of Microbiology, Jawaharlal Nehru Medical College and Hospital, Aligarh, Uttar Pradesh, India over a period of three years from April 2017 to March 2020.

The study was approved by the Institutional Ethics Committee and written informed consent was taken from all patients.

\section{Inclusion Criteria}

Patients of $>15$ years of age having acute febrile illness of more than 5 days with or without acute respiratory failure and acute renal failure (ARF) were included in the study.

\section{Exclusion criteria}

Patients who were $<15$ years of age and those found positive for other febrile illness like typhoid fever, malaria Chikungunya, leptospira, dengue and those with a known underlying etiology relating acute respiratory failure and ARF were excluded from the study.

Clinical profile and presentation was recorded in detail through pre-designed porforma. Clinical grading was also done for assessment of the severity of scrub typhus on the basis of severity scale formulated by Kantipong $\mathrm{P}$ et al. ${ }^{14}$ Patients with uncomplicated scrub typhus were assigned a severity score of zero, while points correspond to the increasing degrees of severity.

Sample collection: $5 \mathrm{ml}$ of blood was collected from suspected patients with undiagnosed fever for serology. Sera were separated by centrifugation at $1512 \times \mathrm{g}$ for 15 minutes and serological tests were performed.

$5 \mathrm{ml}$ of whole blood was collected separately in EDTA vial for DNA extraction by commercially available Qaigen extraction kit. (Qiagen, Hilden, Germany)

\section{Serological testing}

All sera were tested for presence of IgM antibodies for scrub typhus by IgM ELISA (In Bios International Inc. Seattle, WA, USA) and IgM indirect immunofluorescent antibody assay (IFA) (Fuller Laboratories, Fullerton, USA). The results for scrub typhus IgM ELISA were interpreted according to the manufacturer's instructions. A single IFA titer of 1:64 or more was accepted as positive for scrub typhus.

The IFA slides had wells and each well is coated with 4 dots for 4 strains of 0. tsutsugamushi 
for all sample: Boryong, Gilliam, Karp and Kato strains.

A conventional PCR amplification, targeting the 56KDa protein was performed. ${ }^{15}$

\section{Statistical Analysis}

Statistical analysis was conducted on SPSS software (version 20) by Chi-square test. The probability value ( $P$ value) of less than 0.05 was considered significant.

\section{RESULTS}

Around 414 (outpatient and inpatients both) patients were included in the study over three year period. All samples were tested for scrub typhus by IgM ELISA. Of these, $112(27 \%)$ cases were found positive which were further confirmed by IgM IFA. All 112 (27\%) cases showed significant antibody titers and were considered positive for Scrub typhus. Few negative samples were also confirmed by IFA but were tested negative.

Majority of our patients belong to rural areas living in places with nearby bushes. There was preponderance of positive cases during the winter season, peak started from October to January while numbers of positive cases were low during summer and rainy season (Fig. 1).

Among these 112 diagnosed cases of scrub typhus, 59 (52.6\%) were males while 53 (47.3\%) were females. Overall positivity among males was 59 (25.6\%) while in females was 53 (29.1\%) as shown in Table 1. There is no significant difference in positivity for scrub typhus among the two sexes $(P>0.05)$.

Age of patients ranged from 18 years to 74 years. The mean age of scrub typhus positive patients was around $45 \pm 14$ years. Majority of positive cases 90 (80.3\%) were between 1846 years of age. Highest positivity was seen in between $18-32$ years $(55.1 \%)$ of age among both males and females. On statistical analysis, the difference in positivity for scrub typhus in different age group was significant $(P<0.05)$ among both males and females.

Sign and Symptoms were divided into system wise categories: general, gastro-intestinal, renal and respiratory. General and respiratory symptoms were found significantly associated with the disease $(P<0.05)$ while renal symptoms $\&$ and overall, gastro- intestinal involvement was observed to be insignificant statistically $(P>0.05)$. However, among the gastro-intestinal symptoms vomiting and diarrhea were found significantly associated with scrub typhus patients $(P<0.05)$.

The average duration of fever at the time of presentation in our study group was 8 days \pm 2 days. The most common clinical findings were chills $91(81.2 \%)$, myalgia 72 (63.2\%), headache 70 (62.5\%), cough 24 (41.3\%), respiratory distress $23(39.6 \%)$, yellow urine $60(53.5 \%)$, reduction

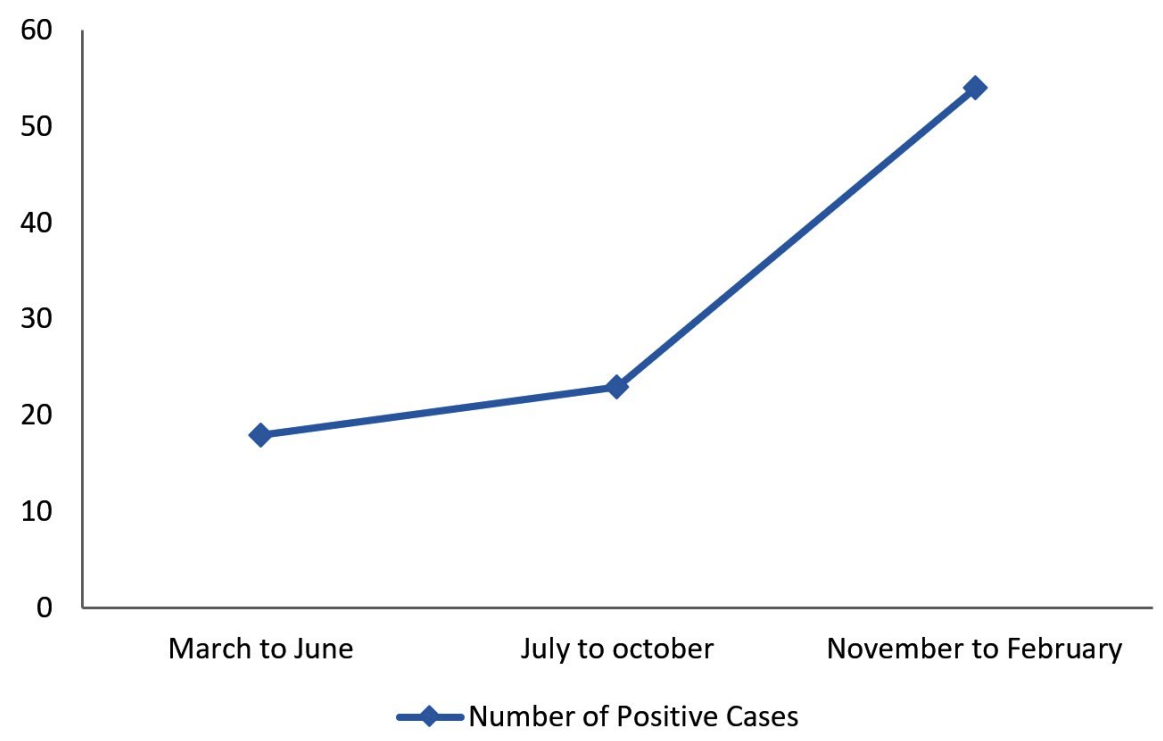

Fig. 1. Month wise positive cases of Scrub typhus from March to February 
in urine output 21 (18.7\%), lymphadenopathy $68(60.7 \%)$ and pain in abdomen $7(12 \%)$ while rashes were present in $2(1.8 \%)$ cases (Table 2 ).

None of the patients diagnosed with scrub typhus had convulsions, mental confusion, gum bleeding, clubbing, cyanosis or epistaxis in our study. Eschar was also not noticed on examination. On clinical grading as per Kantipong scale of severity, none of our positive patient had complications. CNS and cardiac involvement was absent in positive patients while among Gastrointestinal complication, only mild icterus was found in 10 (8.9\%) cases. However, respiratory involvement was there in the form of tachypnoea/ breathlessness giving the score of 1 among 49 (43.7\%) patient while remaining $63(56.2 \%)$ patients had overall score of zero. Fortunately, none of the patients developed severe complication in the form of coma, severe jaundice, and intubation requiring admission. All the positive patients were treated and recovered, hence no mortality was observed.

Gastrointestinal involvement was common in the form of diarrhea $53(47.3 \%)$ and vomiting 58 (51.7\%). Hepatomegaly was not present at the time of examination in our patients. Respiratory complaints were present in nearly $40 \%$ patients, mainly cough was present in $47(41.9 \%)$ patients while tachypnoea and breathlessness was present in 44 (39.3\%) and 49 (43.7\%) respectively. Fortuitously, acute respiratory distress syndrome was not observed in any of the patients.

There was no central nervous system involvement in any of the patient. However, 70
(62.5\%) patients complained of headache. Renal involvement was observed in the form of oliguria 21 (18.7\%) and hematuria 4 (3.5\%).

On comparing the clinical characteristics in scrub typhus positive and negative individuals, it was found that pallor, pain in abdomen, diarrhea, vomiting, lymphadenopathy, tachypnoea were significantly associated with scrub typhus infection ( $P$ value $<0.05$ ).

Unfortunately, we did not get any positive result by PCR. Hence we did not go for strain testing among our positive samples.

\section{DISCUSSION}

Scrub typhus is an acute febrile illness that is widespread in Asia-Pacific region. ${ }^{6}$ Awareness and suspicion of this disease has been neglected for long, especially in our region. This is a hospital based study performed to know the clinical profile and prevalence of scrub typhus among patients monitored during the study period in western Uttar Pradesh.

Total of 414 patients with undiagnosed fever were recruited over two years period. Of these $112(27 \%)$ were found positive for scrub typhus. Our findings are consistent with findings of Behera et al. ${ }^{16}$ In a recent report from New Delhi, they found 34 positive cases out of 314 tested, which is low as compared to prevalence in our study. ${ }^{4}$ Nonetheless, our results are consistent with our previous report, which was a pilot study. ${ }^{13}$ Literature also suggest the varied prevalence of scrub typhus all over India, ranging as low as 3.5\% to as high as $37 \% .{ }^{17,18}$

Table 1. Age and sex wise distribution of febrile cases

\begin{tabular}{|c|c|c|c|c|c|c|}
\hline \multirow{2}{*}{$\begin{array}{l}\text { Age Group } \\
\text { (in years) }\end{array}$} & \multicolumn{3}{|c|}{ Male } & \multicolumn{3}{|c|}{ Female } \\
\hline & Total & Positive(\%) & Negative(\%) & Total & Positive (\%) & Negative (\%) \\
\hline $18-32$ & 58 & $32(55.1)$ & $26(44.8)$ & 49 & $27(55.1)$ & $22(44.8)$ \\
\hline $33-46$ & 58 & $20(34.4)$ & $38(65.5)$ & 42 & $11(26.1)$ & $32(73.8)$ \\
\hline $47-60$ & 62 & $5(8)$ & $12(17.7)$ & 49 & $12(24.4)$ & $37(75.5)$ \\
\hline $61-74$ & 52 & $2(3.8)$ & $50(96.1)$ & 42 & $3(7.1)$ & $39(92.8)$ \\
\hline \multirow[t]{2}{*}{ Total } & 230 & $59(25.6)$ & $172(74.3)$ & 182 & $53(29.1)$ & $130(70.8)$ \\
\hline & & $\begin{array}{c}\text { Chi square } \\
\left(X^{2}\right)=23.5 \\
\text { Degree of } \\
\text { freedom }(\mathrm{df})=1 \\
\mathrm{p}<0.050\end{array}$ & & & $\begin{array}{c}\text { Chi square } \\
\left(X^{2}\right)=14.1 \\
\text { Degree of } \\
\text { freedom }(\mathrm{df})=1 \\
\mathrm{p}<0.050\end{array}$ & \\
\hline
\end{tabular}


Table 2. Clinical manifestations in positive and negative Scrub Typhus cases

\begin{tabular}{|c|c|c|c|c|}
\hline Signs and Symptoms & & $\begin{array}{c}\text { Positive } \\
\text { Cases } \\
(n=112)(\%)\end{array}$ & $\begin{array}{c}\text { Negative } \\
\text { Cases } \\
(n=302)(\%)\end{array}$ & $\begin{array}{c}\text { Test of } \\
\text { significance \& } \\
\text { P-value }\end{array}$ \\
\hline \multirow[t]{6}{*}{ General symptoms } & Chills and rigors & $91(81.2)$ & 195(64.6) & $\begin{array}{c}\left(X^{2}\right)=10.643 \\
(d f)=1, p<0.050\end{array}$ \\
\hline & Rashes & $2(1.8)$ & $6(2)$ & $\begin{array}{c}\left(X^{2}\right)=0.17 \\
(d f)=1, p>0.050\end{array}$ \\
\hline & Headache & $70(62.5)$ & $130(43)$ & $\begin{array}{c}\left(X^{2}\right)=12.38 \\
(d f)=1, p<0.050\end{array}$ \\
\hline & Myalgia & $70(62.5)$ & $158(52.3)$ & $\begin{array}{c}\left(X^{2}\right)=3.423 \\
(d f)=1, p>0.050\end{array}$ \\
\hline & Pallor & $63(56.2)$ & $38(12.6)$ & $\begin{array}{c}\left(X^{2}\right)=84.414 \\
(d f)=1, p<0.050\end{array}$ \\
\hline & Lymphadenopathy & $68(60.7)$ & $38(12.6)$ & $\begin{array}{c}\left(X^{2}\right)=99.364 \\
(d f)=1, p<0.050\end{array}$ \\
\hline \multirow[t]{6}{*}{$\begin{array}{l}\text { Gastro-intestinal } \\
\text { symptoms }\end{array}$} & Vomiting & $58(51.8)$ & $91(30.1)$ & $\begin{array}{c}\left(X^{2}\right)=16.628 \\
(d f)=1, p<0.050\end{array}$ \\
\hline & Diarrhoea & $53(47.3)$ & $65(21.5)$ & $\begin{array}{c}\left(X^{2}\right)=26.683 \\
(d f)=1, p<0.050\end{array}$ \\
\hline & Yellow Urine & $60(53.6)$ & $78(25.8)$ & $\begin{array}{c}\left(X^{2}\right)=28.298 \\
(d f)=1, p<0.050\end{array}$ \\
\hline & Haematemesis & $4(3.6)$ & $26(8.6)$ & $\begin{array}{c}\left(X^{2}\right)=3.085 \\
(d f)=1, p>0.050\end{array}$ \\
\hline & Pain abdomen & 20(17.9) & $29(9.6)$ & $\begin{array}{c}\left(X^{2}\right)=5.335 \\
(d f)=1, p>0.050\end{array}$ \\
\hline & Icterus & $10(8.9)$ & $25(8.3)$ & $\begin{array}{c}\left(X^{2}\right)=0.45 \\
(d f)=1, p>0.050\end{array}$ \\
\hline \multirow[t]{2}{*}{ Renal symptoms } & Oliguria & $21(18.8)$ & $45(14.9)$ & $\begin{array}{c}\left(X^{2}\right)=0.903 \\
(d f)=1, p>0.050\end{array}$ \\
\hline & Haematuria & $4(3.6)$ & $49(16.2)$ & $\begin{array}{c}\left(X^{2}\right)=11.719 \\
(d f)=1, p<0.050\end{array}$ \\
\hline \multirow[t]{3}{*}{ Respiratory symptoms } & Breathlessness & $49(43.8)$ & 39(12.9) & $\begin{array}{c}\left(X^{2}\right)=46.413 \\
(d f)=1, p<0.050\end{array}$ \\
\hline & Tachypnoea & $45(40.2)$ & $40(13.2)$ & $\begin{array}{c}\left(X^{2}\right)=36.324 \\
(d f)=1, p<0.050\end{array}$ \\
\hline & Cough & $46(41.1)$ & $52(17.2)$ & $\begin{array}{c}\left(X^{2}\right)=25.72 \\
(d f)=1, p<0.050\end{array}$ \\
\hline
\end{tabular}

We observed the emergence of positive cases in cluster in the end of October which continued to appear during winters. This is an interesting finding which is similar to report from south India. ${ }^{19}$ While majority of studies, especially in northern India have documented positivity of scrub typhus mainly in rainy season, particularly during the August and September. ${ }^{12,16,17}$

Mean age and other demographic findings of our patients are in concordance with other reports. ${ }^{9,12}$ In our study, there was slight male predominance $(52.6 \%)$ which is consistent with the findings of Varghese et al. ${ }^{9}$

In our study, around 63 (56.2\%) of cases had zero score of severity on Kantipong Scale while $49(43.7 \%)$ had a score of 1 . None of the recent reports discussed this scale. Moreover, Kantipong et al. in their report documented similar findings. ${ }^{14}$ This scale can be used with addition of renal parameters to predict complications and severity of disease. 
We did not observe any eschar among our positive patients. Nearly same finding has been observed in other northern study. ${ }^{4}$ However, south Indian authors reported eschar as a common finding in $55 \%$ of their patients. ${ }^{9,17}$ However, eschar is not a common finding in most literature and cases have been reported even without its presence.

Common clinical findings in the form of vomiting ( $51.7 \%)$, myalgia (63.5\%), cough $(41 \%)$ are in concordance with other report from nearby region. ${ }^{12}$ Other clinical findings such as ( ) are also consistent to other reports from all over India. ${ }^{9,12,18}$ Respiratory involvement was also found significant in this study which has also been confirmed by many researchers. ${ }^{18,20}$

Fortunately, there was no major complications and mortality found in scrub typhus patients. Behera et al. also did not document any mortality among their patients despite the complications in the form of CNS involvement. ${ }^{16}$ Other north Indian studies have reported significant mortality rates among their patients. ${ }^{4,21}$ The reason for low progression to complications and systemic involvement may be because of low virulence of prevailing strains.

Unfortunately, we did not get any positive by conventional PCR. The PCR assay is usually positive only in the first week of disease during the period of rickettsemia. Literature also suggest its low sensitivity beyond the first week of illness ${ }^{4}$ and positivity is low even in nested and real time PCR. ${ }^{4,12}$ Behera et al. got only 3 positive by nested PCR out of 50 samples tested. ${ }^{16}$ The reason for negative results in PCR may be attributed to non availability of eschar material, late presentation of patient beyond first week and the use of conventional technique as literature shows better results of molecular test with nested PCR. ${ }^{4,15}$

Because of the PCR negative findings, conclusive remarks cannot be made on prevalent strain in our region. Varghese et al. documented prevalence of karp and kato like strain in their phylogenetic analysis. ${ }^{9}$ Recently, Chowdhary et al. found similarity of their positive samples with Gilliam. There are very few studies from north depicting prevailing strains in this region. ${ }^{4}$

Despite being a neglected disease, scrub typhus turned out to be a significant etiology of undifferentiated fever. Attitude among clinicians should be changed regarding awareness and suspicion of disease. Scrub typhus should be considered in undiagnosed febrile patients from rural and agricultural background with respiratory and gastrointestinal symptoms. Presence of Eschar is doubtful in our population as we get seropositivity among febrile cases without presence of eschar. Serology turned out reliable in diagnosis of scrub typhus as molecular testing is costly, labor intensive and inconsistent as positivity of molecular test varies with type of sample (eschar or whole blood) and the technique (conventional, nested or real time PCR).

\section{ACKNOWLEDGMENTS}

The authors are thankful to SERB, DST for providing the funds. The authors are also thankful to Mr, Sanjay Sharma, Department of Microbiology, JNMCH, AMU, Aligarh, India for helping with the technical work.

\section{CONFLICT OF INTEREST}

The authors declare that there is no conflict of interest.

\section{AUTHORS' CONTRIBUTION}

AsS conceived, designed the study. FK structured the work order. AfS and CKT conducted experiments. AfS collected the data. AsS wrote the draft manuscript. MR and RC revised the manuscript. All authors read and approved the final manuscript for publication.

\section{FUNDING}

This work was funded by grants from SERB, Department of Science and Technology (DST), INDIA under the Early Career Research Advancement Scheme.

\section{DATA AVAILABILITY}

All datasets generated or analyzed during this study are included in the manuscript.

\section{ETHICS STATEMENT}

This study was approved by the Institutional Ethics and Research Advisory Committee, Aligarh Muslim University, Aligarh, India. 


\section{INFORMED CONSENT}

Written informed consent was obtained from the participants before enrolling in the study.

\section{REFERENCES}

1. Philip CB. Tsutsugamushi disease (scrub typhus) in World War II. Parasitol. 1948;(34):169-191. doi: $10.2307 / 3273264$

2. Chrispal A, Boorugu H, Gopinath KG, et al. Acute undifferentiated febrile illness in adult hospitalized patients: the disease spectrum and diagnostic predictors - an experience from a tertiary care hospital in South India. Trop Doct. 2010;(40):230-234. doi: 10.1258/td.2010.100132

3. World Health Organization. WHO recommended surveillance standards, $2^{\text {nd }}$ ed. No. WHO/CDS/CSR/ ISR/99.2. Geneva: WHO; 2012. https://apps.who.int/ iris/handle/10665/65517

4. Chaudhry R, Thakur CK, Gupta N, et al. Mortality due to scrub typhus - report of five cases. Indian $J$ Med Res. 2019;149(6):790-794. doi: 10.4103/ijmr. IJMR $1314 \_18$

5. Silpapojakul K, Varachit B, Silpapojakul K. Paediatric scrub typhus in Thailand: a study of 73 confirmed cases. Trans R Soc Trop Med Hyg. 2004;98(6):354-359. doi: 10.1016/j.trstmh.2003.10.011

6. Kelly DJ, Fuerst PA, Ching WM, Richards AL. Scrub typhus: the geographic distribution of phenotypic and genotypic variants of Orientia tsutsugamushi. Clin Infect Dis. 2009;48(3):203-230. doi: 10.1086/596576

7. Mittal V, Gupta NK, Bhattacharya D, et al. Serological evidence of rickettsial infections in Delhi. Indian J Med Res. 2012;(135):538-535.

8. Chugh TD. Emerging and re-emerging bacterial diseases in India. J Biosci. 2008;(33):549-555. doi: 10.1007/s12038-008-0073-0

9. Varghese GM, Janardhanan J, Trowbridge P, et al. Scrub typhus in South India: Clinical and laboratory manifestations, genetic variability, and outcome. Int J Infect Dis. 2013;17(11):E981-E987. doi: 10.1016/j. ijid.2013.05.017

10. Batra HV.Spotted fevers and typhus fever in Tamil Nadu. Indian J Med Res. 2007;126:101-103.

11. Mahajan SK, Kashyap R, Kanga A, Sharma V, Prasher BS, Pal LS. Relevance of Weil-Felix test in diagnosis of scrub typhus in India. J Assoc Phys India. 2006;(54):619-621.

12. Rawat V, Singh RK, Kumar A, Saxena SR, Varshney U, Kumar M. Epidemiological, Clinical and Laboratory
Profile of Scrub Typhus Cases Detected by Serology and RT-PCR in Kumaon, Uttarakhand: A HospitalBased Study. Trop Doct. 2018;48(2):103-106. doi: 10.1177/0049475517743891

13. Rizvi M, Sultan A, Chowdhry M, et al. Prevalence of scrub typhus in pyrexia of unknown origin and assessment of interleukin-8, tumor necrosis factor-alpha, and interferon-gamma levels in scrub typhus-positive patients. Indian J Pathol Microbiol. 2018;(61):76-80. doi: 10.4103/IJPM.IJPM_644_16

14. Kantipong P, Watt G, Jongsakul K, Choenchitra C. Infection with Human Immunodeficiency Virus Does Not Influence the Clinical Severity of Scrub Typhus. Clin Infect Dis. 1996;(23):1168-1170. doi: 10.1093/ clinids/23.5.1168

15. Varghese GM, Janardhanan J, Mahajan SK, et al. Molecular Epidemiology and Genetic Diversity of Orientia tsutsugamushi from Patients with Scrub Typhus in 3 Regions of India. Emerg Infect Dis. 2015;21(1)64-69. doi: 10.3201/eid2101.140580

16. Behera B, Biswal M, Das RR, et al. Clinicoepidemiological analysis of scrub typhus in hospitalised patients presenting with acute undifferentiated febrile illness: A hospital-based study from Eastern India. Indian J Med Microbiol. 2019;(37):278-280. doi: 10.4103/ijmm.IJMM_19_147

17. Rao PN, van Eijk AM, Choubey S, et al. Dengue, chikungunya, and scrub typhus are important etiologies of non-malarial febrile illness in Rourkela, Odisha, India. BMC Infect Dis. 2019;19(1):572. doi: 10.1186/s12879-019-4161-6

18. Perumalla SK, Paul S, Abhilash KPP, et al. Eschar and IgM ELISA in the diagnosis of scrub typhus. Indian J Med Microbiol. 2019;37(1):113-115. doi: 10.4103/0255-0857.264495

19. Varghese GM, Raj D, Francis MR, Sarkar R, Trowbridge P, Muliyil J. Epidemiology \& risk factors of scrub typhus in south India. Indian J Med Res. 2016;144(1):76-81. doi: 10.4103/0971-5916.193292

20. Das P, Singh D, Das M, Nayak RK, Mohakud NK. Epidemiological and clinical features of scrub typhus in Odisha, Eastern India. Med J DY Patil Vidyapeeth. 2019;12(5):419-423. doi: 10.4103/ mjdrdypu.mjdrdypu_236_18

21. Bithu R, Kanodia V, Maheshwari RK. Possibility of scrub typhus in fever of unknown origin (FUO) cases: An experience from Rajasthan. Indian J Med Microbiol. 2014;32(4):387-390. doi: 10.4103/0255-0857.142241 